\title{
ARTICLE Succinate-acetate permease from Citrobacter koseri is an anion channel that unidirectionally translocates acetate
}

\author{
Biao Qiu ${ }^{1,2}$, Bingqing Xia ${ }^{3,9}$, Qingtong Zhou ${ }^{4}{ }^{4}$, Yan $\mathrm{Lu}^{1,2}$, Miaomiao He ${ }^{1,2}$, Kazuya Hasegawa ${ }^{5}$, Zhiqiang Ma ${ }^{6}$, Fengyu Zhang ${ }^{7}$, \\ Lichuan $\mathrm{Gu}^{7}$, Qionglei Mao ${ }^{3,9}$, Feng Wang ${ }^{8}$, Suwen Zhao (iD ${ }^{1,4}$, Zhaobing Gao ${ }^{3}$ and Jun Liao (iD ${ }^{1,2}$
}

\begin{abstract}
Acetate is an important metabolite in metabolism and cell signaling. Succinate-Acetate Permease (SatP) superfamily proteins are known to be responsible for acetate transport across membranes, but the nature of this transport remains unknown. Here, we show that the SatP homolog from Citrobacter koseri (SatP_Ck) is an anion channel that can unidirectionally translocate acetate at rates of the order of $\sim 10^{7}$ ions/s. Crystal structures of SatP_Ck in complex with multiple acetates at $1.8 \AA$ reveal that the acetate pathway consists of four acetate-binding sites aligned in a single file that are interrupted by three hydrophobic constrictions. The bound acetates at the four sites are each orientated differently. The acetate at the cytoplasmic vestibule is partially dehydrated, whereas those in the main pore body are fully dehydrated. Aromatic residues within the substrate pathway may coordinate translocation of acetates via anion- $\pi$ interactions. SatP_Ck reveals a new type of selective anion channel and provides a structural and functional template for understanding organic anion transport.
\end{abstract}

Cell Research (2018) 28:644-654; https://doi.org/10.1038/s41422-018-0032-8

\section{INTRODUCTION}

Acetate is a ubiquitous metabolite that is mainly utilized by organisms in the form of acetyl coenzyme A. It is required for both the synthesis and degradation of macromolecules, including proteins, carbohydrates, and fats. ${ }^{1-3}$ Acetate also participates in the regulation of various cellular processes via the acetylation of proteins, ${ }^{4,5}$ such as histone acetylation, ${ }^{6}$ tubulin acetylation, ${ }^{7}$ and p53 acetylation. ${ }^{8}$ In the human digestive tract, acetate comprises more than half of the short-chain fatty acid end products from microbial fermentation, ${ }^{9}$ and this metabolite can be absorbed from the gut into the mammalian circulation system, where it is known to function in multiple signaling mechanisms in hosts including cell proliferation, ${ }^{10}$ secretion of insulin and ghrelin, ${ }^{11,} 12$ and central appetite regulation. ${ }^{13}$

Acetate is an amphipathic monocarboxylate with a hydrophobic methyl tail and a hydrophilic carboxylate head. Several families of transporter proteins in mammals, including proton-linked monocarboxylate transporters (MCTs) and sodium-coupled monocarboxylate transporters (SMCTs), have been shown to non-selectively transport acetate across biological membranes at rates of the order of $\sim 10^{2}-10^{3}$ ions $/ \mathrm{s}^{14-16}$ Previous structural studies on FocA formate channels of the formate-nitrite transporter family have provided for the first time the mechanistic insights into the transport of the formate and other short monocarboxylates. ${ }^{17-20}$ Proteins of the large but poorly characterized
Succinate-Acetate Permease (SatP) superfamily (Supplementary information, Figure S1 and Table S1), which are widespread in bacteria, archaea, fungi and nematodes, are known to be essential for acetate transport. ${ }^{21-23}$ Here, we demonstrate that the SatP homolog from the human pathogenic bacteria Citrobacter koseri (SatP_Ck) is an acetate channel. SatP_Ck translocates acetate in a unidirectional manner at rates of the order of $\sim 10^{7}$ ions $/ \mathrm{s}$. Our crystal structures of SatP_Ck in complex with acetates further uncovered the unique structural basis for this fast and unidirectional translocation.

\section{RESULTS AND DISCUSSION}

SatP_Ck is an anion channel that unidirectionally translocates acetate

Data from cell-based substrate uptake assays with SatP from $E$. coli (SatP_Ec, UniProt primary accession number: P0AC98 ${ }^{24}$ ) from a previous study has been interpreted to mean that this protein is a proton-coupled succinate-acetate transporter. ${ }^{21}$ However, we observed channel behavior for purified SatP_Ck, an ortholog of SatP_Ec that differs by only fifteen residues (Supplementary information, Figure S2), when it was reconstituted into a planar lipid bilayer in electrophysiological experiments. When assayed with a solution containing $50 / 50 \mathrm{mM}$ (trans/cis) chloride and a 20/200 mM (trans/cis) acetate gradient, we observed step-like

\footnotetext{
${ }^{1}$ School of Life Science and Technology, ShanghaiTech University, Shanghai 201210, China; ${ }^{2}$ Institute of Biochemistry and Cell Biology, Chinese Academy of Sciences, 320

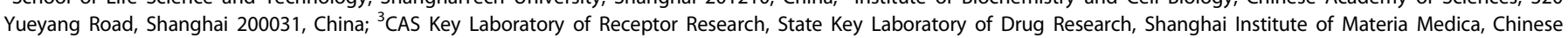

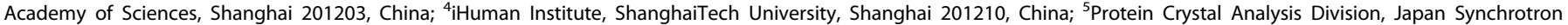

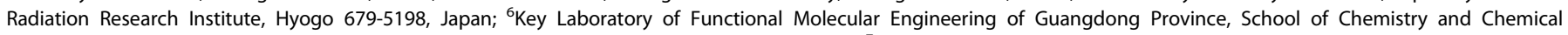

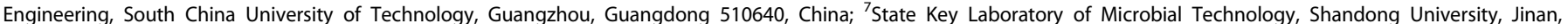

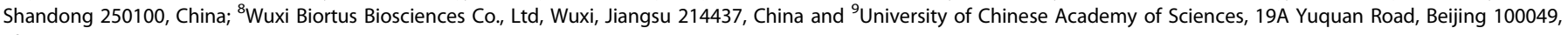
China
}

Correspondence: Zhaobing Gao (zbgao@simm.ac.cn) or Jun Liao (liaojun@shanghaitech.edu.cn)

These authors contributed equally: Biao Qiu, Bingqing Xia.

Received: 3 December 2017 Revised: 8 February 2018 Accepted: 1 March 2018

Published online: 27 March 2018 
currents with single-channel conductance of 102.3 pS (Fig. 1a and Supplementary information, Figure S3a). Moreover, six levels of conductance were recorded in some experiments (Fig. $1 \mathrm{~b}$ and Supplementary information, Figure S4). Presumably, these levels correspond to the openings of one, two, three, four, five, or all six monomeric channels within a given SatP_Ck hexamer, or to separate channels. Although the Nernst equation would predict that the reversal potential of acetate should be approximately $+60 \mathrm{mV}$ with this $20 / 200 \mathrm{mM}$ (trans/cis) gradient, our measured value $(+21.1 \mathrm{mV})$ was considerably lower, suggesting that SatP_Ck can permeate ions besides acetate. When assayed with an equivalent solution that lacked chloride, both the measured currents and the current-voltage plots of SatP_Ck at various acetate gradients revealed that this channel functions as a a

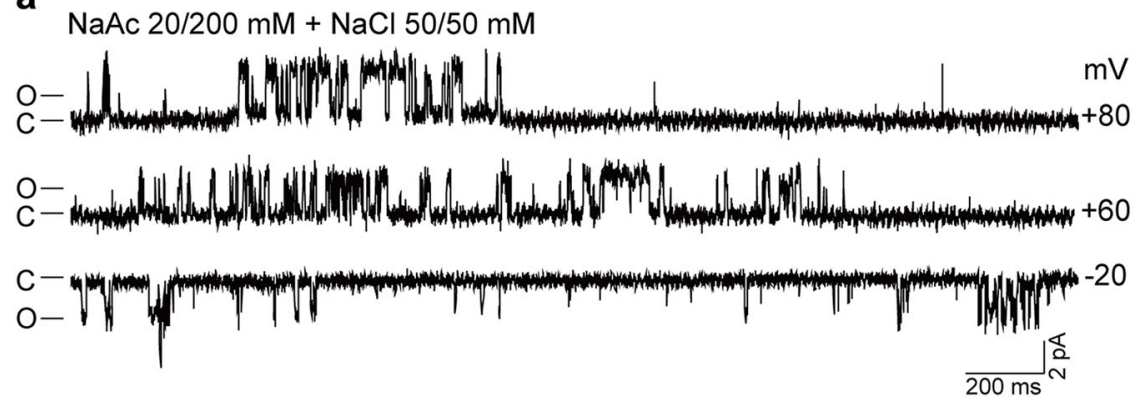

b

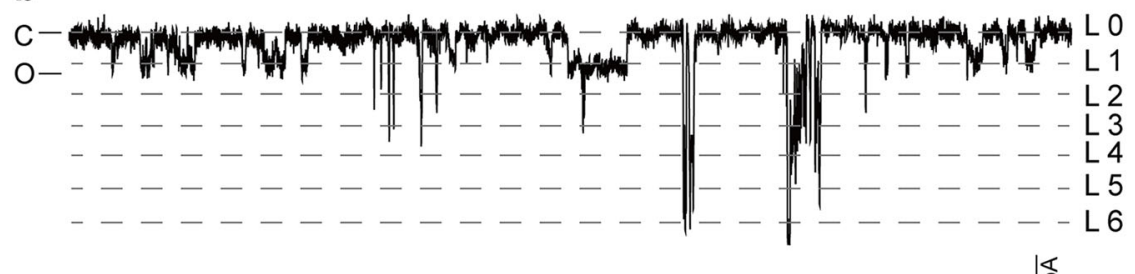

C

$\mathrm{NaAc}$

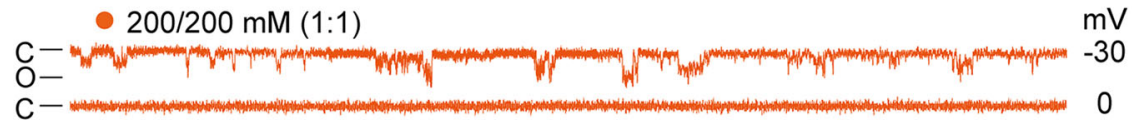

40/200 mM (1:5)

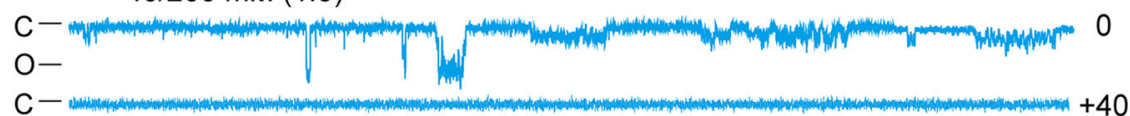

$\triangle 20 / 200 \mathrm{mM}(1: 10)$

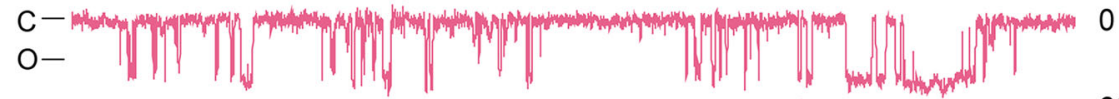

$\mathrm{C}-\mathrm{m}$

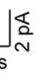
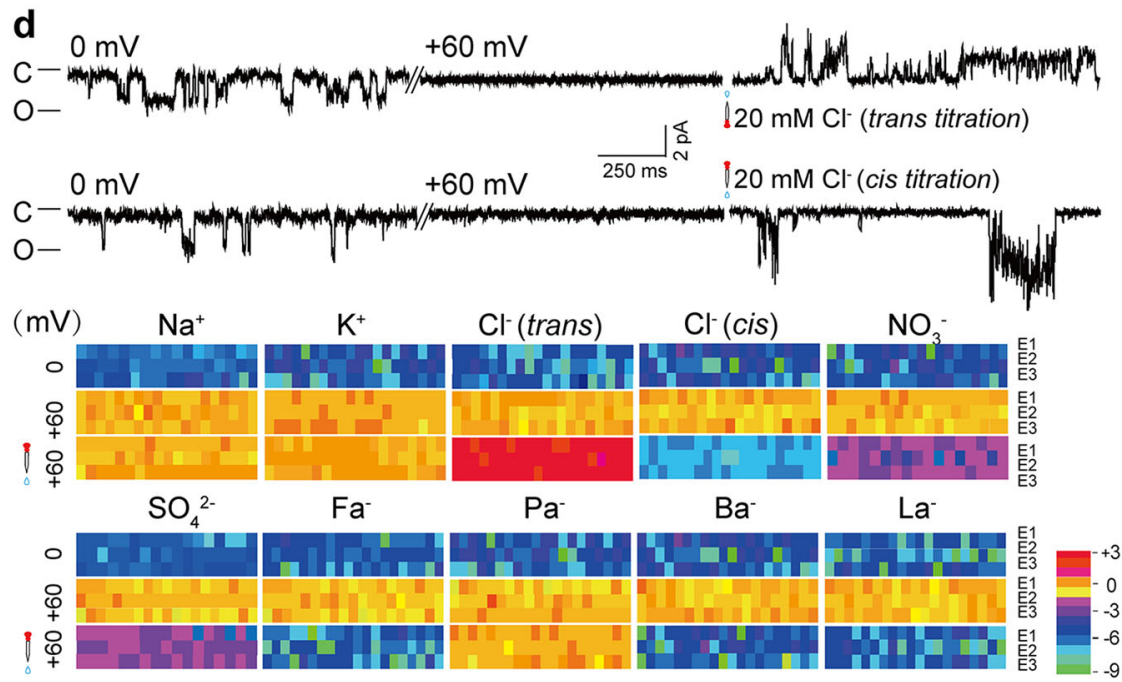
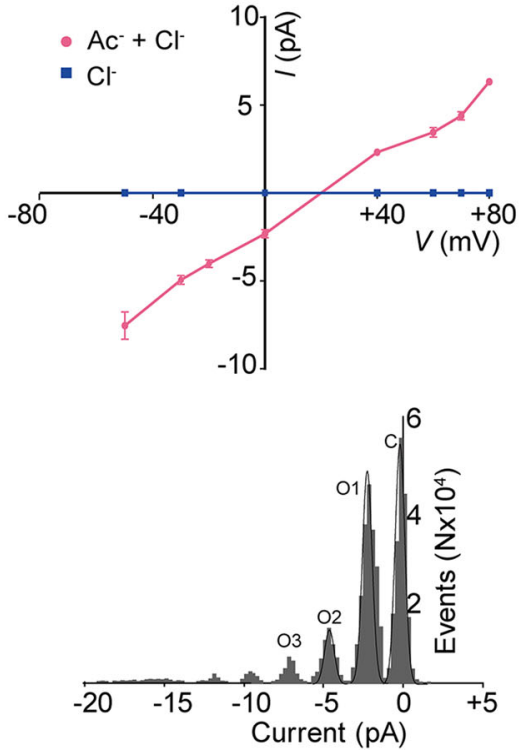

$\mathrm{mV}$
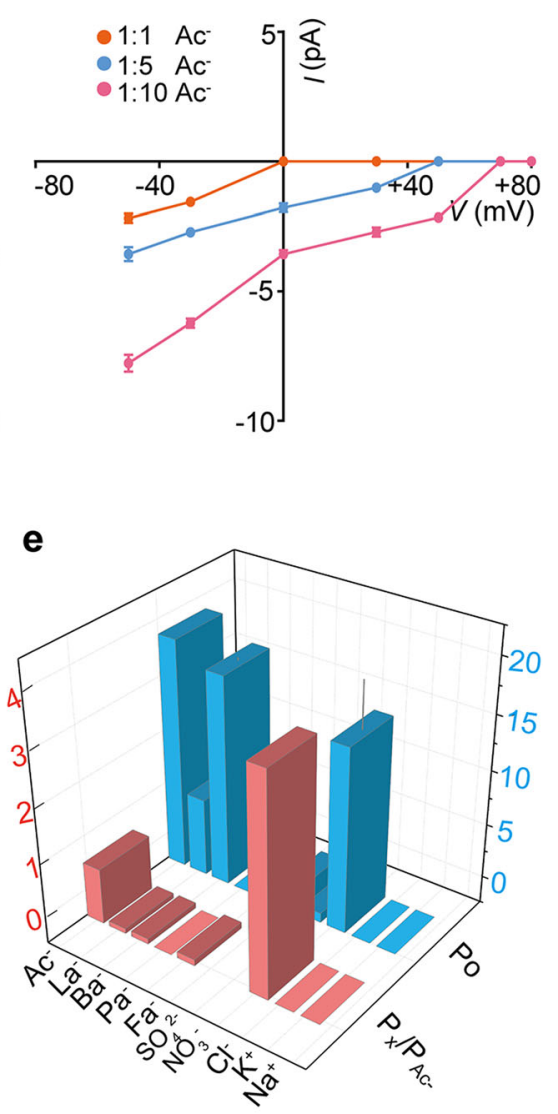
unidirectional conduction channel (i.e., rectifier) at rates of $\sim 10^{7}$ ions/s; no outward current was recorded at the potentials that are higher than the corresponding reversal potentials (Fig. 1c, Supplementary information, Figures S4 and 5).

Ion specificity is typically used to define the various classes of ion channels. To evaluate the ion specificity of SatP_Ck , we conducted both titration assays and classic permeation experiments. In the titration assays, we first eliminated the acetate current at its equilibrium potential of $+60 \mathrm{mV}$ in an asymmetric 20/200 mM (trans/cis) acetate gradient, and then tested different ions individually for their ability to permeate across SatP_Ck by adding them to the cis or the trans side of a lipid bilayer. SatP_Ck is impermeable to cations, such as sodium and potassium. Among the tested anions, SatP_Ck could translocate chloride and other short-chain monocarboxylates, but only in the presence of acetate (Fig. 1a, d; Supplementary information, Figure S3b). In classic permeation experiments, we observed the following sequence of acetate-dependent relative anion permeability: $P_{\text {chloride }}>P_{\text {acetate }}>$
$P_{\text {lactate }}>P_{\text {formate }}>P_{\text {butyrate }}>P_{\text {nitrate }}>P_{\text {sulfate }}{ } P_{\text {propionate }}$ (Fig. 1e and Supplementary information, Figure S3c). Taken together, the results of these electrophysiological experiments demonstrate that SatP_Ck is an acetate channel.

The structural features of SatP_Ck

Lacking any solved structures, previous primary sequence-based bioinformatics studies had proposed two signature motifs for proteins of the SatP superfamily. ${ }^{21,22}$ The first motif (N-P-[AV]-P[LF]-G-L-X-[GSA]-F) contains an aquaporin-like "NPA" stretch. ${ }^{21}$ The second signature motif (S-Y-G-X-F-W) is rich in aromatic residues. ${ }^{22}$ Based on our structures of SatP_Ck, we combined standard primary sequence-based bioinformatics tools and structure-based transmembrane helix assignments to systematically categorize all of the SatP superfamily members in the Pfam database ${ }^{25}$ into three families (Supplementary information, Figures S1 and 2, Table S1). SatP_Ck belongs to Family 1, which comprises $>80 \%$ of the superfamily and contains four subfamilies. All members of Family

a

b

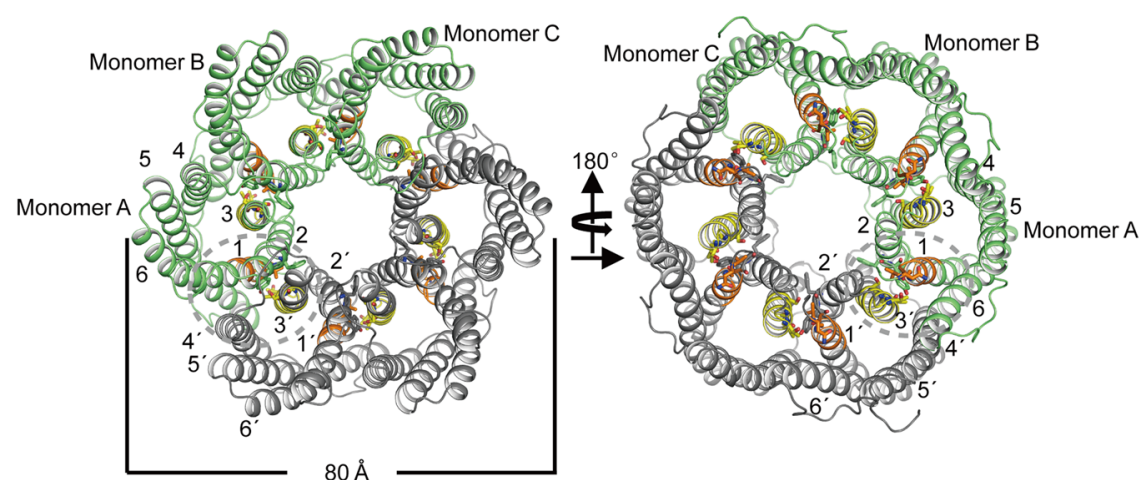

C

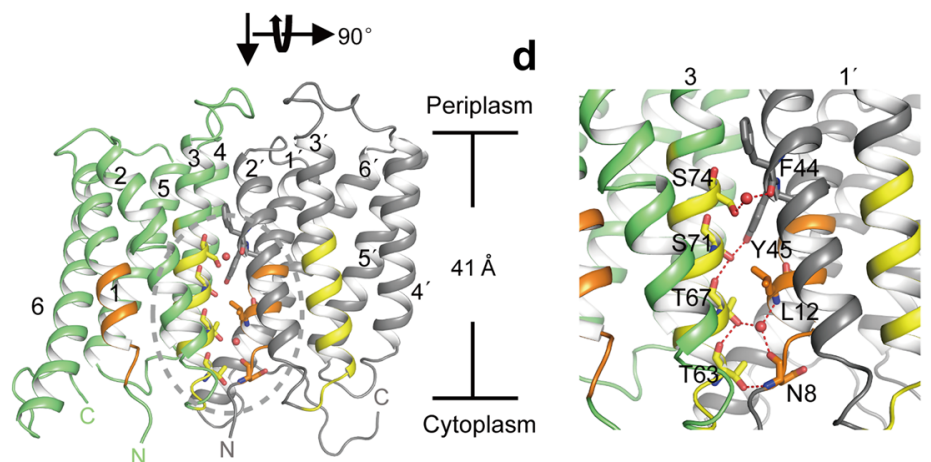

Fig. 2 Structure of the SatP Ck hexamer. a, b Cartoon representations of the SatP Ck hexamer viewed from the periplasmic (a) and cytoplasmic (b) sides. The constituent three monomers of each of the neighboring asymmetric units are colored lime or gray, respectively. TM1 through TM6 are labeled in two adjacent monomers to show their interactions. The dotted circles indicate the inter-monomer interactions involving signature motifs 1 (orange) and 2 (yellow). c Side view of two interacting SatP_Ck monomers. Red spheres represent water molecules, which were removed in $\mathbf{a}$ and $\mathbf{b}$ for clarity. $\mathbf{d}$ A zoom-in view of the inter-monomer hydrogen bonding network

Fig. 1 Electrophysiology of SatP_Ck reconstituted into a planar lipid bilayer. a Single-channel currents at the indicated potentials. Left, representative recordings in a solution containing acetate and chloride. SatP_Ck was added to the cis side, and the membrane potential was clamped at the indicated values (cis side). Right, $I-V$ curves in the same solutions as in the left panel (red) or in the equivalent solution lacking acetate (blue) $(n>20)$. b Macroscopic currents in the same solution as the left panel of a at $0 \mathrm{mV}$. Left, channel opening and closing events, showing six levels of conductance. Right, all-point amplitude histogram for the current trace (bin width $=0.05 \mathrm{pA} / \mathrm{bin}$ ). The conductance was $102.3 \pm 5.5,193.1 \pm 6.7$, and $330.9 \pm 10.3 \mathrm{pS}$ for the one-monomer opened (O1), the two-monomer opened (O2), and the three-monomer opened (O3) states, respectively. ' $C$ ' indicates the closed state. $c$ The unidirectional acetate transport of SatP_Ck. Left, conduction of SatP_Ck at the indicated acetate gradients, at -50 to $+80 \mathrm{mV}$, with acetate as the only permeable anion. Right, the $I-V$ curves derived from the left panel $(n>20)$. d Conduction of different ions in the presence of acetate. Top, representative chloride currents. Bottom, heat maps ${ }^{67}$ of the currents of the indicated ions. 'E1', 'E2', and 'E3' indicate three independent experiments. $\mathrm{K}^{+}$, potassium ion; $\mathrm{Na}^{+}$, sodium ion; $\mathrm{Cl}^{-}$, $\mathrm{chloride}^{-}$ion; $\mathrm{NO}_{3}{ }^{-}$, nitrate ion; $\mathrm{SO}_{4}{ }^{2-}$, sulfate ion; $\mathrm{Ac}^{-}$, acetate; $\mathrm{Ba}^{-}$, butyrate; $\mathrm{Fa}^{-}$, formate; $\mathrm{La}^{-}$, lactate; $\mathrm{Pa}^{-}$, propionate. e Three-dimensional representation of the open probability and the permeability of the indicated ions relative to acetate $(n=4-6)$. All data points represent the mean \pm SEM 
a

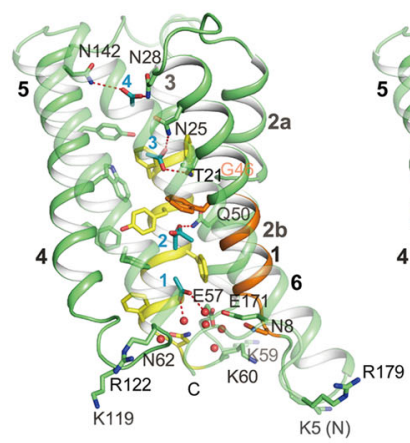

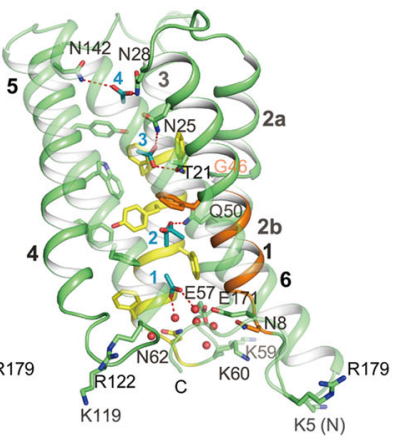

$\mathrm{K5}(\mathrm{N})$

b

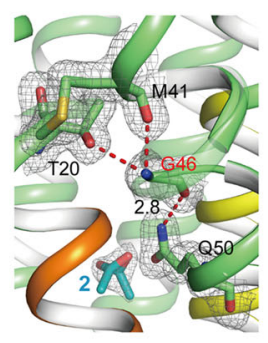

C
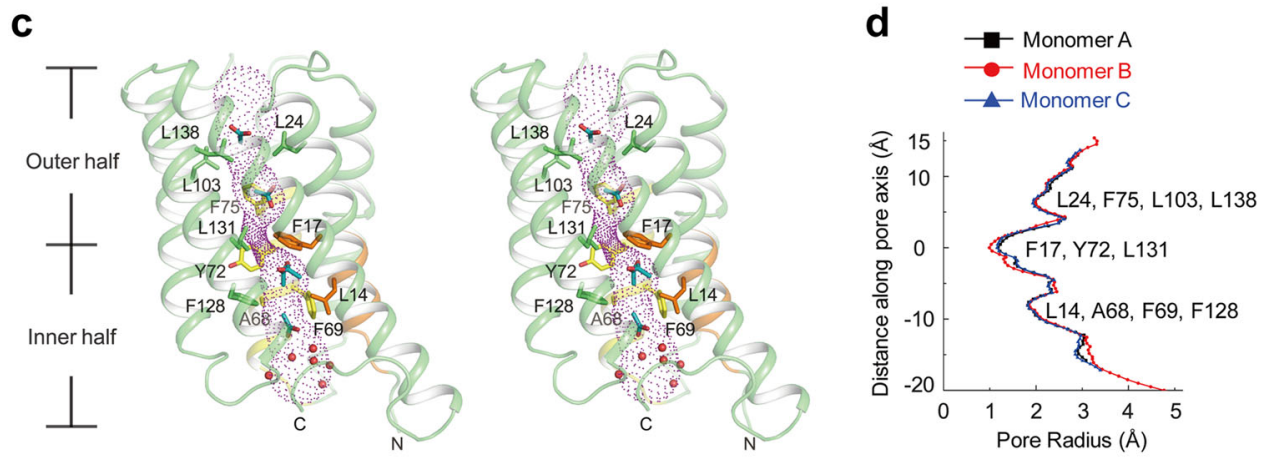

e

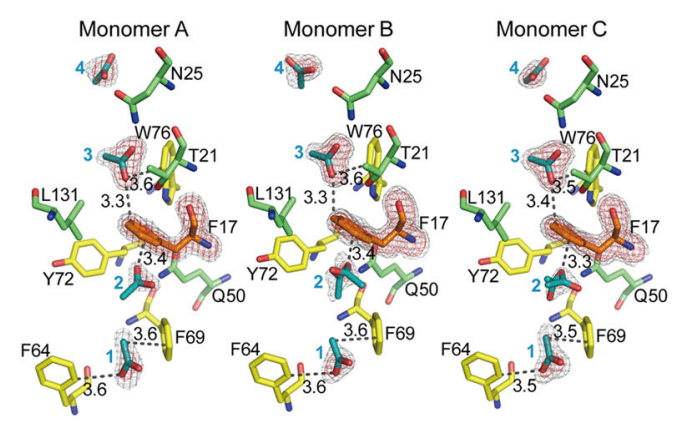

Fig. 3 Structural features of the SatP_Ck monomer and the acetate pathway. a Stereoview of a monomer in ribbon diagram. TM5 and TM6 are drawn transparently to afford a better view of the pathway. The side-chains of charged, polar and aromatic residues lining the pathway, together with the four bound acetates (colored in dark cyan for carbon atoms and red for oxygen atoms), are represented as sticks. $\mathbf{b}$ Q50 can form hydrogen bond with G46. The backbone of G42-A49 is drawn transparently to better show the kink at G46. The 2mFo-DFc density is represented as a gray mesh, contoured at the $1.0 \sigma$ level. c The acetate pathway (shown as purple mesh) and the three hydrophobic constriction sites. d The radii of the acetate pathways along the central pore axes for the three ASU monomers. The origin of each central axis is set at the point of the narrowest central constriction. e Fo-Fc omit map of F17 and the acetates at S1-S4 in each ASU monomer. Gray and red meshes depict electron density as contoured at the 3.0 and or $5.0 \sigma$ levels, respectively. The SatP_Ck structure was obtained at 500 mM acetate concentration

1 contain two highly conserved signature motifs: (1) N-P-[ATG]-PL-G-L-X-[GAS]-F, which is similar to the previously described, and (2) G-[ND]-T-F-[GPAS]-X-[TV]-[AV]-[FL]-X-[SAT]-[YF]-G-X-F-W, which was extended and functionally verified in the present study. SatP Families 2 and 3 members show major distinctions from Family 1 in signature motif 1.

We grew crystals of SatP_Ck in $500 \mathrm{mM}$ acetate with the lipidic cubic phase (LCP) technique. ${ }^{26}$ The crystal structure determined at this high acetate concentration was refined to $1.8 \AA$ resolution (Supplementary information, Table S2). To examine the acetate occupancy at different acetate-binding sites of SatP_Ck, we then slowly replaced the crystallization solutions around the LCP droplets with solutions containing $10 \mathrm{mM}$ acetate once the crystals in $500 \mathrm{mM}$ acetate have reached their full size. The structure determined at this low acetate concentration was refined to $2.8 \AA$. The crystal structures obtained at the two acetate concentrations are virtually identical except for the number of bound acetates. Although our discussion focuses primarily on the high-resolution structure, the lower-resolution structure provides important insights into acetate affinity at the different acetatebinding sites.

The crystals of SatP_Ck are of the $\mathrm{P} 4_{2}$ space group, with three monomers per asymmetric unit (ASU). The three monomers (Monomers $A, B$ and $C$ ) in two neighboring ASUs form a hexamer via a twofold crystallographic symmetry (Fig. 2a, b; Supplementary information, Figure S6a). The contacts among monomers involve not only hydrophobic interactions, but also hydrogen bonds formed primarily among highly conserved residues in the two aforementioned signature motifs (Fig. 2c, d). The strong conservation of residues at these monomer interfaces among the SatP homologs led us to speculate that multimeric assembly is likely common in these proteins, an idea that is consistent with 
prediction from ab initio modeling using evolutionary information. ${ }^{27}$ Of note, studies of SatP homologs in Saccharomyces cerevisiae and Yarrowia lipolytica have suggested the possibility of di- or oligomeric assembly. ${ }^{28}$ Like other anion channels, the central hole of the SatP_Ck hexamer does not function as the anion pathway. ${ }^{17,} 19$

The three SatP_Ck monomers in each crystallographic ASU are essentially identical, with a pairwise R.M.S.D of $0.22-0.27 \AA$ in $C_{a}$ atoms. Each SatP_Ck monomer consists of six transmembrane ahelices (TMs) with both termini at the intracellular side (Figs. 2c, 3a), consistent with the predicted topology for SatP_Ec. ${ }^{21,} 29$ Unlike aquaporin and some other anionic channels, ${ }^{17}, 30,31$ SatP_Ck monomers lack twofold intramolecular pseudosymmetry. TM1 (26 A, 19 residues) starts right after the $\mathrm{N}^{8} \mathrm{PAP}^{11}$ stretch of signature motif 1 and is too short to span the hydrophobic lipid bilayer. F17 of signature motif 1 in monomer B has alternative conformations (Supplementary information, Figure S6b). TM3 and TM4 have a wide inter-helix distance. TM6 is exceptionally long (44 $\AA, \sim 30$ residues), and is oriented at a $\sim 70^{\circ}$ angle to the membrane surface. TM6 helps seal TM1 and the wide inter-helical spacing between TM3 and TM4 of the adjacent monomer (Fig. 2a, b). TM2 is broken into two segments TM2a and TM2b at kinked G46. The backbone carbonyl oxygen of G46 can form hydrogen bond with the side-chain amide nitrogen of Q50 located on TM2b (Fig. 3b). Our studies revealed that the side-chain of Q50 is essential for acetate transport by functioning as a hydrogen bond donor (Supplementary information, Figure S8). Selected residues from the all six TMs together form the acetate pathway. As the structures of FocA channels characterized to date have pentameric assembly with each monomer containing an internal structural repeat, ${ }^{17-20}$ the hexameric architecture of SatP_Ck with monomers lacking intramolecular pseudosymmetry clearly suggests a distinct mechanism(s) for monocarboxylate permeation.

$\mathrm{Ab}$ initio modeling using evolutionary information has predicted that SatP proteins should have similar hexameric assembly and monomeric folding to HpUrel, a proton-gated urea channel from Helicobacter pylori that has been structurally characterized. ${ }^{27}$ We superposed the SatP_Ck monomer with that of HpUrel (PDB code: $3 U X 4)^{32}$ using secondary structure matching algorithm ${ }^{33}$ and revealed R.M.S.D. of $2.5 \AA$ for aligned 144 backbone Ca atoms of the two proteins (Supplementary information, Figure S6c). Despite this overall fold similarity, there are many differences for these two channels, such as the configurations of the constrictions, substrate binding sites and their opposite transmembrane orientations. $^{34}$

Acetate pathway of a SatP_Ck monomer

The intracellular loops immediately outside the cytoplasmic vestibule of the acetate pathway include highly to moderately conserved basic residues (Fig. 3a). These residues may help attract nearby negatively charged acetate anions to cytoplasmic vestibule through electrostatic interaction. The acetate pathway contains four acetate-binding sites (named as S1-S4) that are arranged in a winding, single-file sequence from $\mathrm{S} 1$ at cytoplasmic vestibule to S4 at periplasmic vestibule, with S2 and S3 within the main pore body (Fig. 3a, c). Apart from polar residues at the four acetatebinding sites, the other areas of the acetate pathway are lined by nonpolar residues; many of these are aromatic residues. Each of the pairs of neighboring acetate-binding sites is separated by a hydrophobic constriction. These constrictions are comprised of a

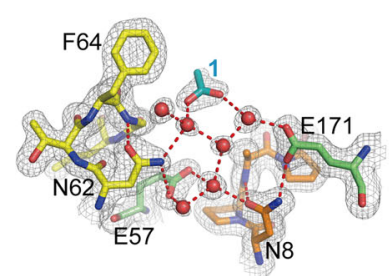

C iad. $\mathrm{MTSET}$

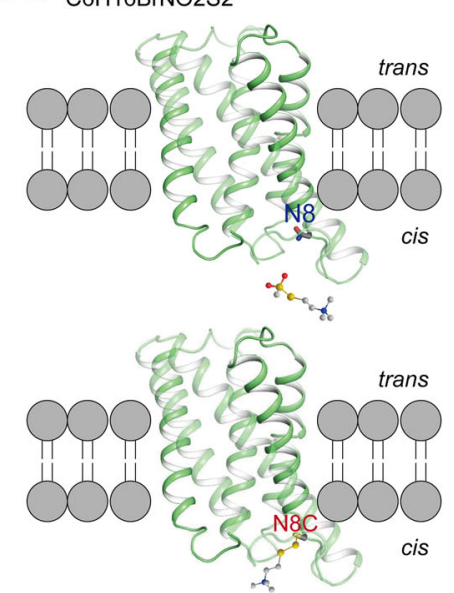

b
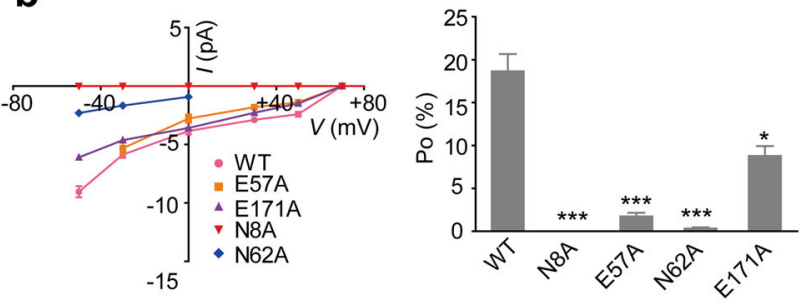
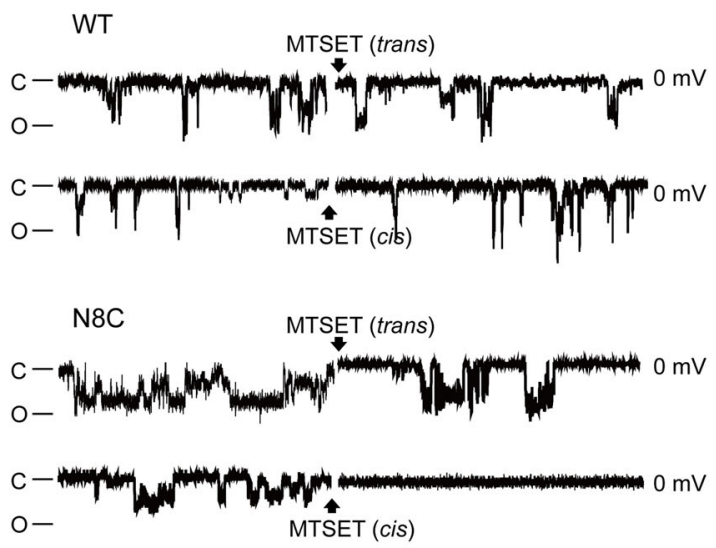

Fig. 4 S1 binds a partially dehydrated acetate. a Hydrogen bonding network at S1. Gray mesh, 2Fo-Fc density contoured at $1.0 \sigma$. b $I-V$ curves (left) and open probabilities (right) for the single alanine mutants of S1 residues in asymmetric acetate solutions (20/200 mM trans/cis) at $0 \mathrm{mV}$. c A cysteine accessibility assay of the mutant N8C of SatP_Ck demonstrating that blockage of S1 at the cis side of the lipid bilayer inhibits the outward passage (inward current) of acetate. Left, the cartoon scheme of the cysteine accessibility assay. MTSET can block accessible cysteine residues and consequently inhibit channel function. Right, wild-type (WT) SatP_Ck channels (Top) were insensitive to MTSET owing to the lack of any accessible cysteine residues. Inward currents of the mutant N8C (Bottom) were inhibited by the administration of MTSET at the cis side of the lipid bilayer $(n=4)$ 
a
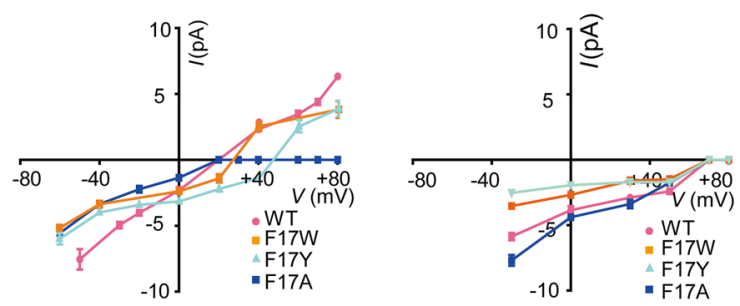

b
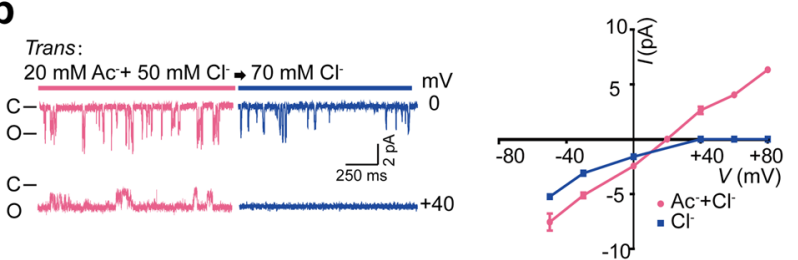

Fig. 5 The impact of $\mathrm{F} 17$ and the $\mathrm{S} 3$ acetate on anion transport. a $1-$ $V$ curves of the mutant variants of F17 in (Left) mixed chloride (50/ $50 \mathrm{mM}$, trans/cis) and acetate $(20 / 200 \mathrm{mM}$, trans/cis) solutions and (Right) asymmetric acetate $(20 / 200 \mathrm{mM}$, trans/cis) solutions $(n>6)$. b The influence of the presence of acetate in the trans side solution on channel permeation behavior. Left, the representative recording of currents (Red) in a solution containing $50 / 50 \mathrm{mM}$ (trans/cis) chloride and 20/200 mM (trans/cis) acetate gradient, and the currents (Blue) after the replacement of acetate by an equal molar concentration of chloride in the trans side solution using a perfusion system at the indicated potentials. Right, $I-V$ curves of SatP_Ck before and after the removal of acetate from the trans side solution $(n>4)$. WT, wildtype SatP_Ck

highly conserved leucine and phenylalanine residues, and more than half of them are located in the two signature motifs. Substrates may therefore undergo a common dehydration mechanism before they can permeate through the main pore of SatP proteins.

The most central and narrowest hydrophobic constriction of the anion pathway, which is formed by $\mathrm{F} 17, \mathrm{Y} 72$, and L131, is only 2.0 $\AA$ in diameter, and this constriction divides the pathway into two sections of similar length $(\sim 15-18 \AA$, Fig. 3c, d). Given that this 2.0 $\AA$ central constriction is physically too narrow to allow the passage of acetates or other smaller anions, we infer that our SatP_Ck crystal structures are in a closed state. Following this, conformational changes involving these residues at the central constriction would be required to shift SatP_Ck to an opened state.

In the SatP_Ck crystal structure obtained in solutions containing $500 \mathrm{mM}$ acetate, S1-S4 are occupied by acetates, with the acetates at S2 and S3 fully dehydrated within the main pore body (Fig. 3a, c, e). The amphipathic acetates appear to "rifle" their way through the acetate pathway, as the acetates at S1-S4 are each orientated differently. The carboxylate head of each acetate forms 1-2 hydrogen bonds with the polar side-chains of pathway residues at S1-S4, whereas the nonpolar methyl tail of acetate face to nonpolar groups (Fig. 3a, c; Supplementary information, Figure S7). With the exception of S4, abolishment of the hydrogen bonding at S1 through S3 via directed mutagenesis significantly decreased acetate conduction (Supplementary information, Figure S8), suggesting an essential role of hydrogen bonding at S1, S2, and S3 for acetate transport.

S1 binds a partially dehydrated acetate

S1 is the only acetate-binding site among the four that binds a partially dehydrated acetate ${ }^{35,} 36$ (i.e., an acetate ion that is less than fully hydrated with water molecules) (Figs. 3a, 4a; Supplementary information, Figures S7 and 9). The acetate is clasped by side-chains of residues N8, N62, E57, and E171 via a watermediated hydrogen bond network. These four polar residues protrude rigidly into the cytoplasmic vestibule of the acetate pathway, with $\mathrm{N} 8$ on the extended $\mathrm{N}^{8} \mathrm{PAP}^{11}$ segment, E57 and N62 on the termini of TM2b and TM3, and E171 on TM6. This protrusion is stable, as the side-chains of N8 and N62 form hydrogen bonds with the nearby backbone atoms of the signature motifs. N8 and N62 further help position E57 and E171 via sidechain to side-chain hydrogen bonding. The positions of N8 and $\mathrm{N} 62$ in the pathway are reminiscent of the well-known asparagine residues in the two NPA motifs of aquaporin. ${ }^{30}$ In aquaporin, these asparagine side-chains are oriented by highly constrained sidechain to main-chain hydrogen bonds to project their ND2 groups strictly into the channel pore and help break proton relay by binding to a central traversing water molecule so that the valences on its oxygen are occupied. ${ }^{30}$

Most water molecules in the hydrogen bond network of S1 each forms three or four hydrogen bonds with their neighboring hydrogen bond acceptors/donors (Supplementary information, Figure S9), mimicking a virtually optimal hydrogen-bonding configuration in aqueous solutions. ${ }^{37}$ Electrophysiological experiments showed that N8A, E57A, and N62A mutants of SatP_Ck lost almost all of their acetate conductivity (Fig. $4 \mathrm{~b}$ and Supplementary information, Figure S8), underscoring the essential roles of these residues in acetate conduction. The importance of S1 for acetate outward translocation is further validated by a cysteine accessibility assay (Fig. 4c). In this assay, the wild-type SatP_Ck and its N8C mutant were separately reconstituted into an artificial lipid bilayer. Then, a membrane-impermeable cysteine modification reagent MTSET ([2-(trimethylammonium) ethyl] methanethiosulfonate bromide) was added to the cis (i.e., S1) or trans (i.e., S4) side of the artificial lipid bilayer. Lacking any accessible endogenous cysteines, wild-type SatP_Ck was insensitive to MTSET. In contrast, the N8C mutant became impermeable to acetate only if MTSET was added to the cis side. Taken together, these results show that $\mathrm{S} 1$ is the site that initiates outward acetate translocation.

The S2 acetate is in close proximity to the S1 acetate and the ring face of F17

It is accepted that ions must overcome a substantial electrostatic barrier to cross-lipid bilayers. ${ }^{38}$ In chloride and fluoride channels, aromatic residues have been highlighted as a key feature conferring halide anion selection and permeation; the positive quadrupolar edges of aromatic rings stabilize negatively charged anions. ${ }^{39-42}$ In SatP_Ck, aromatic rings that populate the anion pathway may function to stabilize acetate anions. Such edgewise interactions between aromatic rings and carboxylate anions have been observed in many protein structures. ${ }^{43,}{ }^{44}$ For example, the highly conserved aromatic residues in each of the signature motifs may interact with acetates at S1 and S3 using edgewise anion- $\pi$ interactions ${ }^{43}, 44$ (Fig. 3e and Supplementary information, Figure S7): the acetate at S1 is stabilized by an edgewise interaction with F64 ( 3.5-3.6 $\AA)$; the acetate at S3 is stabilized by edgewise interactions with both F17 ( 3.3-3.4 $\AA$ ) and W76 ( 3.5-3.6 $\AA$ ). The attractive anion- $\pi$ interactions of anions with the faces of electrondeficient arenes are widely observed. ${ }^{45}$ We noticed that the carboxylate of the S2 acetate is underneath the face of F17's aromatic ring $(\sim 3.3-3.4 \AA)$. However, it is difficult to determine whether the anion- $\pi$ interaction of the S2 acetate with the face of F17's aromatic ring is attractive or repulsive, since benzyl group is not electron-deficient. We also observed alternative conformations of the S2 acetate in our structures: its carboxylate plane can be roughly parallel or roughly vertical to the aromatic ring plane of F17. Regardless of which conformation it assumes, the S2 acetate remains in close proximity to both the $\mathrm{S} 1$ acetate and the face of F17's aromatic ring (Fig. 3e and Supplementary information, Figure S7). The steric repulsions between the two acetates at S1 and S2 and between the acetate at S2 and F17 will force F17 to undergo a conformational change when $\mathrm{S} 1$ and $\mathrm{S} 2$ are filled by acetates during outward translocation. 
F17 and the S3 acetate together impact the direction of anion transport

F17 is strongly conserved among SatP Family 1 proteins (Supplementary information, Figure S2), and is located at the aforementioned narrowest constriction site, underscoring its functional importance. Our electrophysiology experiments with F17 mutants in mixed chloride and acetate solutions indicated that the aromatic ring of F17 regulates anion transport (Fig. 5a). The replacement of phenylalanine with physiochemically similar aromatic residues (F17W, F17Y) does not change the bidirectional permeation behavior of SatP_Ck for a mixed acetate and chloride solution. The F17A mutant, however, exhibits only unidirectional anion permeation, regardless of the solution contents. These results revealed the sophisticated interactions that occur among the aromatic ring of F17, chloride, and acetate during anion transport.

Although these observations do not by themselves reveal the precise mechanism(s) underlying the directionality of anion transport, some insights can be gleaned from the atomic structure of SatP_Ck in complex with acetates. In particular, for outward acetate translocation to occur, the aromatic ring of F17 must presumably rotate or swing to widen the central constriction. The aromatic ring of F17 is located close to the side-chains of T21 $(\sim 3.3 \AA)$ and W76 ( 3.5 ̊) (Supplementary information, Figure S7). Such steric effects imply that there are cooperative conformational changes among the side-chains of F17, T21, and W76. Moreover, since our structure shows that T21 stabilizes the S3 acetate via hydrogen bonding, whereas F17 and W76 stabilize the S3 acetate via attractive anion- $\pi$ interactions, the cooperative conformational changes that disrupt these interactions would reduce the affinity of acetate at $\mathrm{S} 3$ and therefore ease the movement of the acetate at S2 toward S3.

The S3 acetate remains at its binding site in the crystal structures obtained at an acetate concentration of $10 \mathrm{mM}$ (Supplementary information, Figure S10). At this low acetate concentration, the other three acetate-binding sites are no longer consistently occupied by acetates. Lacking an apo structure, we were unable to determine whether the presence of the $\$ 3$ acetate is essential for maintaining the structure of outer half of the pathway. However, we noticed functional importance of the S3 acetate in a perfusion experiment in which the acetate in an acetate/chloride solution on the trans side of a lipid bilayer was gradually removed (Fig. 5b). We expected that the removal of acetate from the trans side should accelerate the disassociation of the acetate from S3, since the overall binding affinity of acetate to the channel is known to be in the milli-molar range. ${ }^{21}$ Consistent with this speculation, we found that the removal of acetate from the trans side solution altered the permeation behavior of SatP Ck: the bidirectional permeation of anions through SatP Ck became unidirectional, and adding the acetate back restored bidirectional permeation. This change suggests that the acetate at S3 at least partially regulates the directionality of the anion permeation. It is thus clear that the S3 acetate and the aromatic ring of F17 together impact the directionality of anion translocation.

It is notable that SatP_Ck could translocate chloride and other short-chain monocarboxylates, but only in the presence of acetate (Fig. 1a, d; Supplementary information, Figure S3b). One plausible explanation for the observed necessity of acetate is that the presence of acetate may have influence on SatP_Ck opening (e.g., keeps the channel in conductive states). Such permeant ion effects on channel gating have been observed in many other channels. $^{46-48}$

A possible role of stepwise dehydration in unidirectional acetate translocation

Our SatP Ck structure drew our attention to an important distinction about the solvation status of acetates as they proceed through S1 at cytoplasmic vestibule to S4 at periplasmic vestibule during outward translocation. Namely, the acetate at $S 1$ is partially dehydrated, whereas the acetates at S2 and S3 inside main body of pore are fully dehydrated. At S4, the acetate appears to be positioned for energetically-favorable rehydration. In classic tetrameric cation channels (e.g., $\mathrm{K}^{+}, \mathrm{Na}^{+}$and $\mathrm{Ca}^{2+}$ channels), ions usually go through so-called selectivity filters via a dehydration step, resulting in ion-dependent free-energy barriers and thus giving rise to selectivity for particular ions. ${ }^{49-51}$ Under physiological conditions, the hydration energy of an acetate anion is known to be 10 times higher than that of an acetic acid molecule, owing to the stronger hydrogen bonds formed between the anion and water molecule. ${ }^{35,36,52}$ The ability of S1 at the cytoplasmic vestibule of the pathway to bind a partially dehydrated acetate anion creates a two-step dehydration process that has major energetic implications for the function of SatP_Ck. Considerably more energy would be required to fully dehydrate the substrate in a single step. By separating the dehydration process into two steps (binding to S1, subsequently binding to S2), SatP_Ck attenuates the amount of energy required for initiating acetate transport. No such multi-step attenuation mechanism has been observed for other structurally characterized anion channels. The presence of such a mechanism in SatP_Ck is not unreasonable when one considers that an organic anion like acetate has a relatively more complicated hydration structure than an inorganic anion, owing to its more complex distribution of electronegativity.

In conclusion, SatP_Ck represents a new type of anion selective channel. Our study revealed possible principles underlying the unidirectional transport of amphipathic acetate across membranes. A simplified transport process of acetate through SatP_Ck is drawn in Fig. 6: positively charged, basic residues in the intracellular loops of SatP_Ck help draw acetates towards the cytoplasmic vestibule. The loading of a partially dehydrated acetate to S1 initiates translocation process. The steric repulsions between the two acetates at S1 and S2 and between the acetate at S2 and F17 trigger a conformational change in F17 that opens the main pore. Cooperative conformational changes in residues spatially adjacent to F17 then weaken the binding affinity of the acetate at S3, pushing an outgoing acetate towards S4. Finally, the energetically-favorable rehydration of the acetate at S4 drives substrate release from the anion pathway.

\section{MATERIALS AND METHODS}

Bioinformatics analysis of the SatP superfamily The Enzyme Function Initiative-Enzyme Similarity Tool (EFI-EST) ${ }^{53}$ was used to generate a sequence similarity network (Supplementary information, Figure S1) for the SatP Pfam superfamily PF01184 (http://pfam.xfam.org/family/PF01184, 5,113 members, 3,433 unique sequences). Using an $E$-value cutoff of $e^{-30}$, the biggest cluster (denoted as Family 1) has 2,826 unique sequences, covering archaea (159 sequences), bacteria (787 sequences), and eukaryotes (1,865 sequences), as well as 15 environmental sequences (Supplementary information, Table S1). Other notable clusters at $\mathrm{e}^{-30}$ are Families 2 and 3 . Family 1 can be further divided into 4 subfamilies (1A-1D) at $e^{-55}$. It should be noticed that the above classification is not based on functional data. To find the signature motifs, we filtered Family 1 by discarding sequences that do not have all six predicted transmembrane helices. After this filtering, 2,388 non-redundant sequences remained. We then conducted a multiple sequence alignment for these sequences using MAFFT L-INS-I. ${ }^{54}$ Sequence logos were created with WebLogo ${ }^{55}$ using the aligned fragments covering each transmembrane helix. Finally, the sequences N-P-[ATG]-P-LG-L-X-[GAS]-F and G-[ND]-T-F-[GPAS]-X-[TV]-[AV]-[FL]-X-[SAT]-[YF]G-X-F-W were defined as signature motifs 1 and 2, respectively, based on the current functional and structural understanding generated in studies of SatP_Ck. 
a
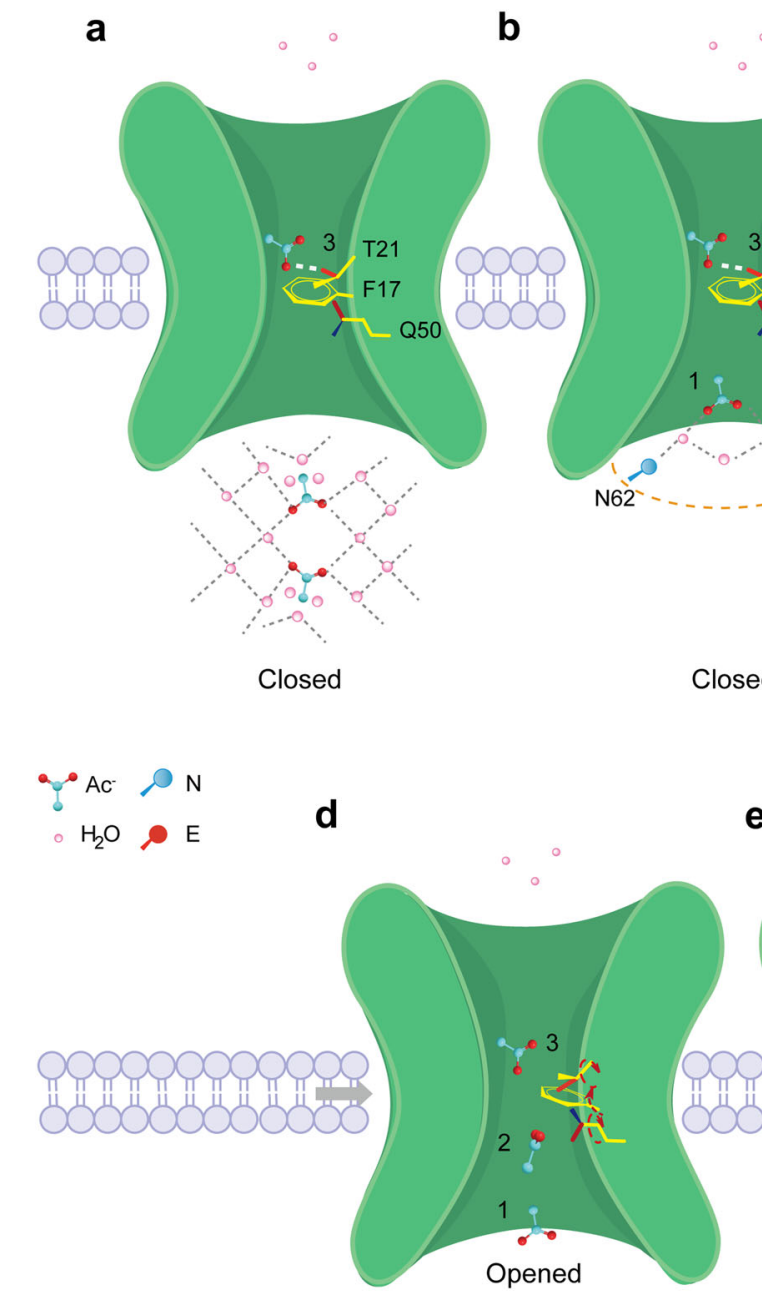

Closed
C
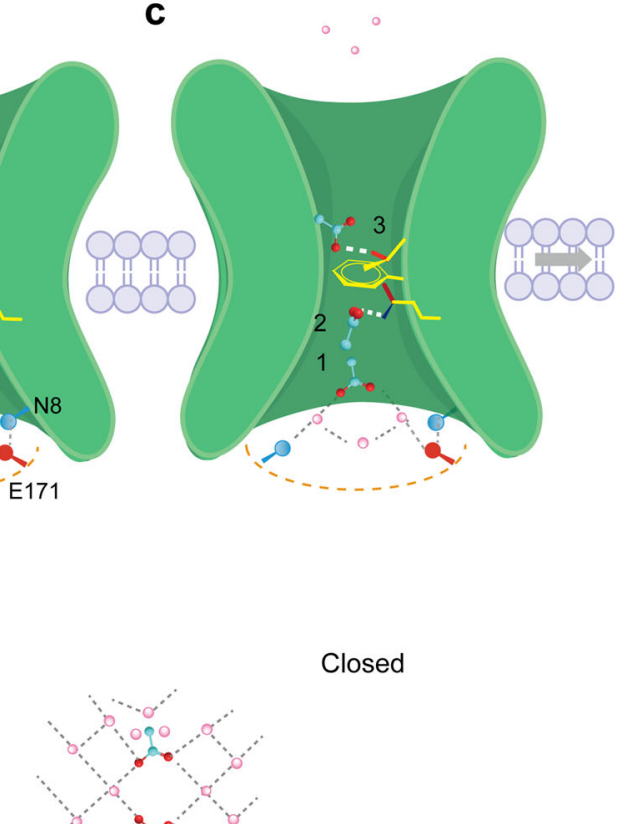

Closed

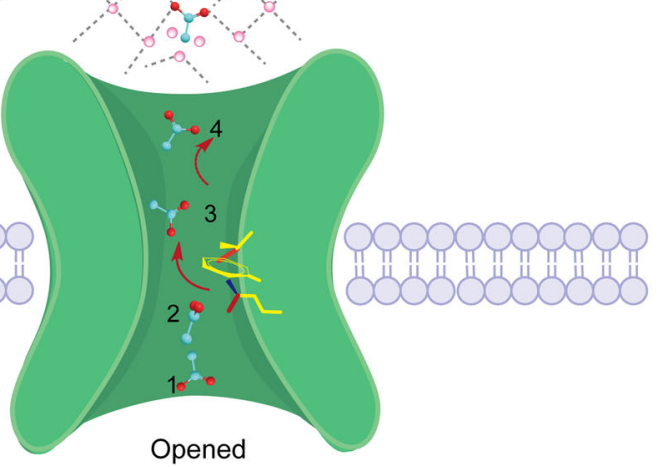

Fig. 6 Schematic drawing of the representative stages of the unidirectional acetate translocation process. a The channel is in a closed state with S3 bound with a dehydrated acetate. b The channel is in a closed state with S1 and S3 bound, respectively, with a partially dehydrated acetate and a dehydrated acetate. c The channel is in a closed state with S1 bound with a partially dehydrated acetate and S2 and S3 bound with dehydrated acetates. Steric repulsions between the acetates at S1 and S2 and between the acetate at S2 and the aromatic ring of F17 trigger conformational changes in F17 and its adjacent residues. $\mathbf{d}$ The channel is in an opened state, following conformational changes of F17 and its adjacent residues. The binding affinity of acetate at S3 is weakened by the aforementioned conformational changes in F17 and its adjacent residues. e The channel is in an opened state with S3 now occupied by an acetate that has moved there from S2. S4 is occupied by an acetate that is released from S3

\section{Protein expression and purification}

The genes encoding full-length SatP (SatP Ck) from Citrobacter koseri ATCC BAA-895 and its mutants were individually subcloned into the PQE60 vector (Qiagen) between the Ncol and BamHI restriction sites, followed by a thrombin cleavage sequence (LVPRGS) and a hexahistidine ( $\mathrm{His}_{6}$ ) tag. E. coli M15 cells (Qiagen) containing the individual recombinant plasmid described above were cultured at $37^{\circ} \mathrm{C}$ in $20 \mathrm{~L}$ of Luria-Bertani liquid medium to $A_{600}=\sim 0.6$; protein expression was induced with $0.4 \mathrm{mM}$ isopropyl $\beta$-D-thiogalactoside (IPTG). After overnight growth at $18^{\circ} \mathrm{C}$, cells expressing the target protein were harvested and homogenized with an EmusiFlex-C3 homogenizer (Avestin) in solution containing $50 \mathrm{mM}$ HEPES ( $\mathrm{pH} 7.3$ ), $100 \mathrm{mM} \mathrm{NaAc}$ and $50 \mathrm{mM} \mathrm{NaCl}$ with addition of $10 \mathrm{mM}$ phenylmethanesulfonyl fluoride (PMSF) and $0.1 \mathrm{mg} / \mathrm{mL}$ DNAse (Sigma). After removal of cell debris by centrifugation at $9,000 \times \mathrm{g}$ for $15 \mathrm{~min}$ at $4{ }^{\circ} \mathrm{C}$, the membrane fraction was harvested from the supernatant by centrifugation at $100,000 \times g$ for $1 \mathrm{~h}$ at $4{ }^{\circ} \mathrm{C}$. The protein was then extracted from the collected membrane fraction by incubating with $40 \mathrm{mM} n$-Decyl$\beta$-D-Maltopyranoside (DM, Anatrace) in the same solution for $2 \mathrm{~h}$ at room temperature. After another centrifugation at $25,000 \times g$ for
$30 \mathrm{~min}$ at $4{ }^{\circ} \mathrm{C}$, the supernatant was loaded onto a Talon $\mathrm{Co}^{2+}$ affinity column (Clontech) pre-equilibrated with solution containing $20 \mathrm{mM}$ HEPES ( $\mathrm{pH}$ 7.3), $100 \mathrm{mM} \mathrm{NaAc}, 50 \mathrm{mM} \mathrm{NaCl}$ and $4 \mathrm{mM}$ $\mathrm{DM}$, followed by a wash of the column with the equilibration solution containing $30 \mathrm{mM}$ imidazole. The protein was eluted with the equilibrium solution containing $300 \mathrm{mM}$ imidazole. The eluate was further treated with thrombin (Sigma) at one unit per $3 \mathrm{mg}$ of protein overnight at room temperature to remove the hexahistidine tag. The digested proteins were further concentrated to 25 $\mathrm{mg} / \mathrm{mL}$ and purified by size-exclusion chromatography using a Superdex-200 (10/300 GL) column (GE Healthcare) in 20 mM MES (pH 6.5), $100 \mathrm{mM} \mathrm{NaAc}$ and $4 \mathrm{mM}$ DM. The peak fraction of the wild-type SatP_Ck between 10.3 and $12.4 \mathrm{~mL}$ was collected and concentrated to about $40 \mathrm{mg} / \mathrm{mL}$ for the LCP crystallization. The peak fraction of each SatP_Ck mutant eluted within the same volume range $(10.3-12.4 \mathrm{~mL})$ was stored at $4{ }^{\circ} \mathrm{C}$ for further functional studies.

Protein crystallization

Wild-type SatP_Ck was crystallized with the LCP technique. ${ }^{26,56}$ Briefly, concentrated SatP_Ck was first reconstituted into 1-(7Z- 
652

pentadecenoyl)-rac-glycerol (7.8 MAG, Avanti) with a protein/lipid weight ratio of 1:1.2 with the two-syringe method. ${ }^{26}$ Crystals were screened and optimized in Corning 96-well crystallization plates by overlaying $5 \mu \mathrm{L}$ of crystallization solutions over $70 \mathrm{~nL}$ of protein-laden LCP drops using a sitting drop method. Crystals grew to a final size of $100 \times 100 \times 15 \mu \mathrm{m}$ in 1 week in a solution containing 0.1 M MES (pH 6.5), 0.1 M NaAc, 0.2 $\mathrm{M} \mathrm{CaAc}_{2}$ and $~ 45 \%$ PEG400 (v/v). These crystals have high quality diffraction up to 1.8 $\AA$ A. Heavy atom-derivative crystals were prepared by replacing $2 \mu \mathrm{L}$ of the overlaid crystallization solution with $2 \mu \mathrm{L}$ of the same solution containing $10 \mathrm{mM} \mathrm{K}_{2} \mathrm{OsO}_{4}$, upon the appearance of fullsize native crystals. The crystals soaked in this solution for 2-3 days gave good anomalous signals. To further check the affinity of acetates inside the SatP_Ck channel passage, we equilibrated the native crystal in crystallization solution at varied acetate concentrations. Notably, crystals soaked in acetate concentrations lower than $10 \mathrm{mM}$ had dramatically deteriorated diffraction quality, suggesting that the further removal of acetate may destabilize the protein or the crystal packing. The SatP_Ck crystals in $10 \mathrm{mM}$ acetate were obtained using the following protocol: $2-3 \mu \mathrm{L}$ of crystallization solutions overlaying proteinladen LCP droplets were gradually replaced by the same volume of solution containing $0.1 \mathrm{M}$ MES (pH 6.5), $10 \mathrm{mM} \mathrm{NaAc}, 90 \mathrm{mM}$ $\mathrm{NaCl}, 0.2 \mathrm{M} \mathrm{CaCl}_{2}$ and $45 \%$ PEG400 (v/v). This same replacement of solution was repeated 6-10 times (each time followed by an overnight equilibration) until the $\mathrm{NaAc}$ in the crystal drops reached the targeted concentration. All crystals were frozen directly in liquid nitrogen.

Data collection, structure determination, and refinement

The native and the anomalous scattering data sets for crystals obtained in $500 \mathrm{mM}$ acetate were collected at beamline BL41XU at the Spring-8 facility in Japan with $0.97900 \AA$ and $1.13970 \AA$ wavelengths. These data sets were further processed and scaled using HKL3000. ${ }^{57}$ The structure was determined by the method of single isomorphous replacement with anomalous scattering (SIRAS) using osmium derivative crystals. Osmium sites were determined using SHELXD, ${ }^{58}$ and initial phases were improved using Crank. ${ }^{59}$ Model building and refinement were iteratively carried out using PHENIX ${ }^{60}$ and Coot. ${ }^{61}$ The final model of SatP_Ck was refined to $1.8 \AA$ with $R_{\text {work }}=16.2 \%$ and $R_{\text {free }}=19.7 \%$, and contained three protein monomers $(A, B$, and $C)$ per asymmetric unit, with the substrate pathway of each monomer bound by four acetates. Monomer A has residues 5-188; and the C-terminal LVPR residues were retained after thrombin cleavage. Monomers $B$ and C contain residues 5-186; in both of these monomers, each acetate at the S2 binding site inside the pathway adopted alternative conformations. The refined structure has 98.9 and $1.1 \%$ of residues, respectively, in the most favorable and additionally allowed regions in the Ramachandran plot. The diffraction data sets for crystals obtained in $10 \mathrm{mM}$ acetate were collected at $1.0 \AA$ at the BL41XU of Spring-8 and beamlines BL17U1, BL18U1, and BL19U1 of the Shanghai Synchrotron Radiation Facility. The data sets were further processed and scaled using XDS. ${ }^{62}$ The structure was determined by the molecular replacement method using the program PHASER ${ }^{63}$ with the $1.8 \AA$ structure as the search model, followed by repeated cycles of model building with Coot and refinement with PHENIX. The final model was refined to $2.8 \AA$. The first five residues at the $\mathrm{N}$-terminus and the last one or two residues at the $\mathrm{C}$-terminus of each monomer in the final model were missing owing to flexibility. The refined structure has $98.5 \%$ and $1.5 \%$ of residues in the most favorable region and additionally allowed region, respectively, on the Ramachandran plot. Detailed crystallographic data and refinement statistics for crystal forms at $500 \mathrm{mM}$ and $10 \mathrm{mM}$ acetate are shown in Supplementary information, Table S2. The pore radii of the anion pathways in three independent monomers $(A, B, C)$ were analyzed using the Caver program. ${ }^{64}$ The electrostatic potentials were calculated by
Poisson-Boltzmann ESP program of Schrodinger with the default parameters and the electrostatic potential surfaces were generated by the program Maestro. ${ }^{65}$ All water molecules and acetate anions have been removed for the calculation of electrostatic potentials. Other structure figures were prepared with PyMOL. ${ }^{66}$ The structure of monomer B (obtained at $500 \mathrm{mM}$ acetate concentration) is used for the cartoon representation in all figures, unless otherwise specified.

\section{Planar bilayer lipid membrane recording}

The currents of purified wild-type SatP_Ck and its mutants were recorded with a voltage-clamp mode using a Warner BC-535 bilayer clamp amplifier (Warner Instruments) filtered at $1-2 \mathrm{kHz}$, $25^{\circ} \mathrm{C}$. The single-channel currents were recorded under the voltage range from $-50 \mathrm{mV}$ to $+90 \mathrm{mV}$ and digitized using pCLAMP 10.2 software (Molecular Devices). The macroscopic currents were measured under the voltage range from $-40 \mathrm{mV}$ to $+100 \mathrm{mV}$ or $-80 \mathrm{mV}$ to $+120 \mathrm{mV}$. Lipid bilayers were prepared using lipoid E80 (Lipoid $\mathrm{GmbH}$ ). All solutions were buffered by 10 mM HEPES ( $\mathrm{pH}$ 6.9). The single-channel conductance and dwell times were determined by fitting to Gaussian functions and exponential equations (single- or bi-), respectively. Opening times less than $1.5 \mathrm{~ms}$ were ignored.

To examine the ability of different ions to pass through SatP_Ck, a titration experiment was used to isolate pure currents. After the protein was added to the asymmetric acetate solutions (20/200 $\mathrm{mM}$, trans/cis), the frequent and continuous inward acetate currents indicated that SatP_Ck was successfully incorporated into the lipid bilayer. The membrane potential was then changed to the equilibrium potential of acetate, $\sim+60 \mathrm{mV}$, to eliminate the acetate currents. Next, the solutions containing $20 \mathrm{mM}$ (final concentration) of the analyte ion were added to the trans or cis side. If the addition of one type of ion induces step-like currents, it demonstrates that SatP_Ck is permeable to this type of ion. Heat maps were plotted using the $\mathrm{R}$ programming language. Each point of a single-channel recording was represented with different colors, blue for inward currents and red for outward currents. To calculate the permeability of different anions relative to acetate, the macroscopic channel currents in the mixed solutions of acetate and $\mathrm{NaX}(X=$ chloride, formate, butyrate, etc.) were recorded. The relative permeability of each anion was deduced based on the shift of the reversal potential in classic assays. The theoretical equilibrium potential and the ion permeability were calculated using the Nernst and Goldman-Hodgkin-Katz flux equations, respectively. The open probability $P_{\mathrm{o}, i}=\tau_{\mathrm{o}}, \mathrm{i} / \mathrm{T}$, where $\tau_{\mathrm{o}}, \mathrm{i}$ is the total time that the channel was observed in the open state and $T$ is the total recording time.

A cysteine accessibility assay was conducted with the N8C mutant of SatP_Ck using a previously described protocol with minor modifications. ${ }^{67}$ Briefly, residue N8 of SatP_Ck was substituted with cysteine to make the N8C mutant. Wild-type SatP_Ck does not contain any accessible endogenous cysteine residues. Inward currents of wild-type SatP_Ck or the N8C mutant were measured using an asymmetric acetate solution $(20 / 200 \mathrm{mM}$, trans/cis) at $0 \mathrm{mV}$, and their sensitivity to a membraneimpermeable cysteine modification reagent MTSET (Biotium Hayward) was examined individually. Analyte proteins for which the current was not altered after administration of MTSET were categorized as insensitive; proteins for which the current was immediately inhibited after the administration of MTSET and for which this current was not restored in 10 min were categorized as sensitive. MTSET was prepared freshly and was added to a bulk solution at the cis or trans side of the lipid bilayer at a final concentration of $100 \mu \mathrm{M}$.

To study the influence of trans acetate on chloride translocation, a perfusion system was used to change the trans side solutions. Briefly, after the recording of typical step-like currents in acetate/ 
chloride mixed solutions that validated the successful incorporation of active SatP_Ck into the planar lipid bilayer, the trans side solutions were gradually replaced with solutions containing 70 $\mathrm{mM}$ sodium chloride. The volume of the trans side chamber was 1 $\mathrm{mL}$, and the volume of perfused $\mathrm{NaCl}$ solution was more than 8 $\mathrm{mL}$, with a flow rate of $2 \mathrm{~mL} / \mathrm{min}$.

All chemicals were of analytical grade or higher, and were purchased from Sigma unless stated otherwise. Data are presented as the mean \pm SEM. All statistical analyses were performed using Student's $t$-tests (GraphPad Prism 5 Software). Asterisks $\left(^{*}\right)$ indicate statistically significant differences from the control group $\left({ }^{*} P<0.05,{ }^{* *} P<0.01\right.$, and $\left.{ }^{* * *} P<0.001\right)$.

\section{Accession codes}

The atomic coordinates and structural factors have been deposited in the Protein Data Bank with the accession numbers $5 Y S 3$ and 5 YS8.

\section{ACKNOWLEDGEMENTS}

The synchrotron radiation experiments were performed at BL41XU of the Spring-8 facility with the approval of the Japan Synchrotron Radiation Research Institute (JASRI) (proposal numbers 2015B1933, 2014B1158, 2015A1100, 2015B2100, 2016A2531, 2016B2531, and 2017A2559), and beamlines BL17U1, BL18U1, and BL19U1 of the Shanghai Synchrotron Radiation Facility (SSRF). This work was supported by the Ministry of Science and Technology of China (2017YFA0504800), the National Science and Technology Major Projects for "Major New Drugs Innovation and Development" (2018ZX09711003-003-003), the National Key Scientific Instrument and Equipment Development Program (2012YQ03026010), and the Joint Fund of the National Natural Science Foundation of China and the Israel Science Foundation Research Program (8146114802). B.X. was supported by the China PostDoctoral Science Foundation (2016M600344). We also thank the Shanghai Municipal Government and ShanghaiTech University for financial support.

\section{AUTHORS CONTRIBUTIONS:}

B.Q. cloned constructs for structure determination, purified proteins, and crystallized SatP_Ck. B.Q. and J.L. collected and analyzed diffraction data, solved the crystal structures, and built and refined atomic models. F.W. and Z.M. participated in crystal optimization. K.H. participated in diffraction data collection. B.Q., Y.L., M.H., F.Z., and L. G. performed mutagenesis. B.X., Q.M., and G.Z. performed channel recordings and analysis. Q.Z. and S.Z. did bioinformatics analysis. J.L. and Z.G. wrote the manuscript with inputs from all authors.

\section{ADDITIONAL INFORMATION}

Supplementary information accompanies this paper at https://doi.org/10.1038/ s41422-018-0032-8.

Competing interests: The authors declare no competing financial interests.

\section{REFERENCES}

1. Randle, P. J., Garland, P. B., Hales, C. N. \& Newsholme, E. A. The glucose fatty-acid cycle. Its role in insulin sensitivity and the metabolic disturbances of diabetes mellitus. Lancet 281, 785-789 (1963).

2. Baldwin, J. E. \& Krebs, H. The evolution of metabolic cycles. Nature 291, 381-382 (1981).

3. Kornberg, H. L. \& Krebs, H. A. Synthesis of cell constituents from C2-units by a modified tricarboxylic acid cycle. Nature 179, 988-991 (1957).

4. Kamphorst, J. J., Chung, M. K., Fan, J. \& Rabinowitz, J. D. Quantitative analysis of acetyl-CoA production in hypoxic cancer cells reveals substantial contribution from acetate. Cancer Metab. 2, 23 (2014).

5. Choudhary, C., Weinert, B. T., Nishida, Y., Verdin, E. \& Mann, M. The growing landscape of lysine acetylation links metabolism and cell signalling. Nat. Rev. Mol. Cell Biol. 15, 536-550 (2014).

6. Allfrey, V. G., Faulkner, R. \& Mirsky, A. E. Acetylation and methylation of histones and their possible role in the regulation of RNA synthesis. Proc. Natl Acad. Sci. USA 51, 786-794 (1964)

7. L'Hernault, S. W. \& Rosenbaum, J. L. Chlamydomonas alpha-tubulin is posttranslationally modified by acetylation on the epsilon-amino group of a lysine. Biochemistry 24, 473-478 (1985)
8. Gu, W. \& Roeder, R. G. Activation of p53 sequence-specific DNA binding by acetylation of the p53 C-terminal domain. Cell 90, 595-606 (1997).

9. Cummings, J. H., Pomare, E. W., Branch, W. J., Naylor, C. P. \& Macfarlane, G. T. Short chain fatty acids in human large intestine, portal, hepatic and venous blood. Gut 28, 1221-1227 (1987).

10. Le Poul, E. et al. Functional characterization of human receptors for short chain fatty acids and their role in polymorphonuclear cell activation. J. Biol. Chem. 278, 25481-25489 (2003).

11. Perry, R. J. et al. Acetate mediates a microbiome-brain- $\beta$-cell axis to promote metabolic syndrome. Nature 534, 213-217 (2016).

12. Canfora, E. E., Jocken, J. W. \& Blaak, E. E. Short-chain fatty acids in control of body weight and insulin sensitivity. Nat. Rev. Endocrinol. 11, 577-591 (2015).

13. Frost, $G$. et al. The short-chain fatty acid acetate reduces appetite via a central homeostatic mechanism. Nat. Commun. 5, 3611 (2014).

14. Halestrap, A. P. \& Price, N. T. The proton-linked monocarboxylate transporter (MCT) family: structure, function and regulation. Biochem. J. 343, 281-299 (1999).

15. Ganapathy, V. et al. Sodium-coupled monocarboxylate transporters in normal tissues and in cancer. AAPS J. 10, 193-199 (2008).

16. Garcia, C. K., Goldstein, J. L., Pathak, R. K., Anderson, R. G. W. \& Brown, M. S. Molecular characterization of a membrane transporter for lactate, pyruvate, and other monocarboxylates: Implications for the Cori cycle. Cell 76, 865-873 (1994).

17. Wang, Y. et al. Structure of the formate transporter FocA reveals a pentameric aquaporin-like channel. Nature 462, 467-472 (2009).

18. Waight, A. B., Love, J. \& Wang, D. N. Structure and mechanism of a pentameric formate channel. Nat. Struct. Mol. Biol. 17, 31-37 (2010).

19. $\mathrm{Lu}, \mathrm{W}$. et al. pH-dependent gating in a FocA formate channel. Science 332 352-354 (2011).

20. Lü, W. et al. The formate channel FocA exports the products of mixed-acid fermentation. Proc. Natl Acad. Sci. USA 109, 13254-13259 (2012).

21. Sa-Pessoa, J. et al. SATP $(\mathrm{YaaH})$, a succinate-acetate transporter protein in Escherichia coli. Biochem. J. 454, 585-595 (2013)

22. Augstein, A., Barth, K., Gentsch, M., Kohlwein, S. D. \& Barth, G. Characterization, localization and functional analysis of Gpr1p, a protein affecting sensitivity to acetic acid in the yeast Yarrowia lipolytica. Microbiology 149, 589-600 (2003).

23. Casal, M., Queirós, O., Talaia, G., Ribas, D. \& Paiva, S. In Yeast Membrane Transport (José Ramos, Hana Sychrová, \& Maik Kschischo eds) 229-251 (Springer International Publishing, 2016).

24. Consortium, T. U. UniProt: the universal protein knowledgebase. Nucleic Acids Res. 45, D158-D169 (2017).

25. Finn, R. D. et al. The Pfam protein families database: towards a more sustainable future. Nucleic Acids Res. 44, D279-D285 (2016).

26. Caffrey, M. \& Cherezov, V. Crystallizing membrane proteins using lipidic mesophases. Nat. Protoc. 4, 706-731 (2009).

27. Ovchinnikov, S. et al. Large-scale determination of previously unsolved protein structures using evolutionary information. elife 4, e09248 (2015).

28. Gentsch, M., Kuschel, M., Schlegel, S. \& Barth, G. Mutations at different sites in members of the Gpr1/Fun34/YaaH protein family cause hypersensitivity to acetic acid in Saccharomyces cerevisiae as well as in Yarrowia lipolytica. FEMS Yeast Res. 7, 380-390 (2007)

29. Daley, D. O. et al. Global topology analysis of the Escherichia coli inner membrane proteome. Science 308, 1321-1323 (2005).

30. Agre, P. et al. Aquaporin water channels-from atomic structure to clinical medicine. J. Physiol. 542, 3-16 (2002).

31. Dutzler, R., Campbell, E. B., Cadene, M., Chait, B. T. \& MacKinnon, R. X-ray structure of a $\mathrm{CIC}$ chloride channel at 3.0 A reveals the molecular basis of anion selectivity. Nature 415, 287-294 (2002)

32. Strugatsky, D. et al. Structure of the proton-gated urea channel from the gastric pathogen Helicobacter pylori. Nature 493, 255-258 (2012).

33. Krissinel, E. \& Henrick, K. Secondary-structure matching (SSM), a new tool for fast protein structure alignment in three dimensions. Acta Crystallogr. D Biol. Crystallogr. 60, 2256-2268 (2004)

34. Strugatsky, D. et al. Structure of the proton-gated urea channel from the gastric pathogen Helicobacter pylori. Nature 493, 255-258 (2013).

35. Fedotova, M. V. \& Kruchinin, S. E. Hydration of acetic acid and acetate ion in wate studied by 1D-RISM theory. J. Mol. Liq. 164, 201-206 (2011).

36. Naganuma, H., Kameda, Y. \& Usuki, T. Neutron and X-ray diffraction studies on the structure of concentrated aqueous sodium acetate solutions (Proceedings of the 1st International Symposium on Advanced Science Research (ASR-2000), Advances in Neutron Scattering Research). J. Phys. Soc. Jpn. 70, 356-358 (2001).

37. Nelson, D. L., Lehninger, A. L. \& Cox, M. M. Lehninger principles of biochemistry. (Macmillan, 2008).

38. Parsegian, V. A. Ion-membrane interactions as structural forces. Ann. N. Y. Acad. Sci. 264, 161-171 (1975).

39. Kane Dickson, V., Pedi, L. \& Long, S. B. Structure and insights into the function of a $\mathrm{Ca}(2+)$-activated $\mathrm{Cl}(-)$ channel. Nature 516, 213-218 (2014). 
40. Yang, T. et al. Structure and selectivity in bestrophin ion channels. Science 346, 355-359 (2014)

41. Stockbridge, R. B. et al. Crystal structures of a double-barrelled fluoride ion channel. Nature 525, 548-551 (2015).

42. Park, E., Campbell, E. B. \& MacKinnon, R. Structure of a CLC chloride ion channel by cryo-electron microscopy. Nature 541, 500-505 (2017).

43. Philip, V. et al. A survey of aspartate-phenylalanine and glutamate-phenylalanine interactions in the protein data bank: searching for anion- $\pi$ pairs. Biochemistry $\mathbf{5 0}$, 2939-2950 (2011).

44. Jackson, M. R. et al. A preference for edgewise interactions between aromatic rings and carboxylate anions: the biological relevance of anion-quadrupole interactions. J. Phys. Chem. B 111, 8242-8249 (2007).

45. Giese, M., Albrecht, M. \& Rissanen, K. Experimental investigation of anion-[small pi] interactions - applications and biochemical relevance. Chem. Commun. 52, 1778-1795 (2016).

46. Holmgren, M. Influence of permeant ions on gating in cyclic nucleotide-gated channels. J. Gen. Physiol. 121, 61-72 (2003).

47. Pusch, M., Ludewig, U., Rehfeldt, A. \& Jentsch, T. J. Gating of the voltagedependent chloride channel CIC-0 by the permeant anion. Nature 373, 527 (1995).

48. Neyton, J. \& Pelleschi, M. Multi-ion occupancy alters gating in high-conductance, $\mathrm{Ca}(2+)$-activated K + channels. J. Gen. Physiol. 97, 641-665 (1991).

49. Doyle, D. A. et al. The structure of the potassium channel: molecular basis of $\mathrm{K}^{+}$ conduction and selectivity. Science 280, 69-77 (1998).

50. Tang, L. et al. Structural basis for $\mathrm{Ca}^{2+}$ selectivity of a voltage-gated calcium channel. Nature 505, 56-61 (2014).

51. Zhou, Y., Morais-Cabral, J. H., Kaufman, A. \& MacKinnon, R. Chemistry of ion coordination and hydration revealed by a $\mathrm{K}^{+}$channel-Fab complex at $2.0 \AA$ resolution. Nature 414, 43 (2001).

52. Payaka, A., Tongraar, A. \& Rode, B. M. Combined QM/MM MD study of HCOO (-)-water hydrogen bonds in aqueous solution. J. Phys. Chem. A 113, 3291-3298 (2009).
53. Gerlt, J. A. et al. Enzyme function initiative-enzyme similarity tool (EFI-EST): a web tool for generating protein sequence similarity networks. Biochim. Biophys. Acta 1854, 1019-1037 (2015)

54. Katoh, K. \& Standley, D. M. MAFFT multiple sequence alignment software version 7: improvements in performance and usability. Mol. Biol. Evol. 30, 772-780 (2013).

55. Crooks, G. E., Hon, G., Chandonia, J.-M. \& Brenner, S. E. WebLogo: a sequence logo generator. Genome Res. 14, 1188-1190 (2004).

56. Liao, J. et al. Structural insight into the ion-exchange mechanism of the sodium/ calcium exchanger. Science 335, 686-690 (2012).

57. Minor, W., Cymborowski, M., Otwinowski, Z. \& Chruszcz, M. HKL-3000: the integration of data reduction and structure solution - from diffraction images to an initial model in minutes. Acta Crystallogr. D Biol. Crystallogr. 62, 859-866 (2006).

58. Sheldrick, G. A short history of SHELX. Acta Crystallogr. A 64, 112-122 (2008).

59. Ness, S. R., de Graaff, R. A. G., Abrahams, J. P. \& Pannu, N. S. Crank. Structure 12, 1753-1761 (2004).

60. Adams, P. D. et al. PHENIX: building new software for automated crystallographic structure determination. Acta Crystallogr. D Biol. Crystallogr. 58, 1948-1954 (2002).

61. Emsley, P., Lohkamp, B., Scott, W. G. \& Cowtan, K. Features and development of Coot. Acta Crystallogr. D Biol. Crystallogr. 66, 486-501 (2010).

62. Kabsch, W. Integration, scaling, space-group assignment and post-refinement. Acta Crystallogr. D Biol. Crystallogr. 66, 133-144 (2010).

63. McCoy, A. J. et al. Phaser crystallographic software. J. Appl. Crystallogr. 40, 658-674 (2007).

64. Petřek, M. et al. CAVER: a new tool to explore routes from protein clefts, pockets and cavities. BMC Bioinform. 7, 316 (2006).

65. Schrödinger Release 2017-1: Maestro, Schrödinger, LLC, New York, NY, 2017. (2017).

66. DeLano, W. L. The PyMOL Molecular Graphics System. (DeLano Scientific, San Carlos, CA, USA, 2002. http://www.pymol.org

67. Xia, B. et al. MLKL forms cation channels. Cell Res. 26, 517-528 (2016). 